a concise description of each species. This includes the local name or names (if any); its distribution in Australia; its status-whether stationary or migratory, comparative abundance, \&c. ; the kind of country it frequents; a short description of its size and plumage, and a few words as to its song or other notes, and its food. All the species are illustrated, and in the majority of cases are figured in colours as well as in black and white. The illustrations are, with few exceptions, from specimens in the National Museum.

In addition to this useful and necessary, but somewhat dry, portion of the handbook, about a third of the little volume is occupied by a most interesting lecture on the Australian avifauna. Thus the book appeals to a much wider class of naturalists than that for which it has been mainly written. For the ornithologists of other countries will find in it an excellent introduction to, and a valuable account of, the birds of a very interesting part of the world. Mr. Tate in his introduction alludes to the growth of a generation trained to look upon the characteristic beauties of Australia with an appreciation almost unknown to their pioneering fathers and mothers, and he combats the popular belief that their birds are songless. An index to the coloured plates and a general index make reference to any particular bird easy.

Unity in Nature: an Analogy between Music and

Life. By C. E. Stromeyer. Pp. $\mathrm{x}+58 \mathrm{~g}$. (London and Manchester: Sherratt and Hughes, Igrr.) Price I2s. 6 d. net.

THrs is a readable discourse on things in general, from physics and astronomy to ethics and politics. As the title indicates, the author expounds certain musical analogies, such as the relation between intervals in the octave and distances in the solar system; but, after the first few sections, the matter of the book becomes more general. There is a good deal of amusingly-put speculation about the kind of world that a "flatland" of two dimensions would be (as sketched by Mr. Hinton), and this, of course, leads to fourth-dimensional space and what might happen there. Then, after a chapter on sexual ethics in which a more or less Schopenhauerian doctrine is taught-with much apt illustration, historical and geographical we come to the female suffrage question, on which the author has vigorous opinions. If women get the vote, "there is every probability that female Members of Parliament would soon be elected; these would decide to elect female Prime Ministers, and as Parliament claims to be omnipotent, there is the prospect of having autocratic female rulers" (p. 507). Also on the disproportionate number of lawyers in Parliament Mr. Stromeyer has some cutting and probably justified remarks; and on education he enters a wise protest against too much classicism. The punctuation of the book leaves something to be desired, and on p. I04 "bromide" appears several times when "bromine" is meant; but these are small details. The author shows wide culture and has a pleasant style.

No. 22 I3, vor. 89]
LETTERS TO THE EDITOR.

[The Editor does not hold himself responsible for opinions expressed by his correspondents. Neither can he undertake to return, or to correspond with the writers of, rejected manuscripts intended for this or any other part of NaTURE. No notice is taken of anonymous communications.]

Prof. Bergson and the Eye of Pecten.

I FIND that Prof. Bergson in his Philosophies has been making use of a comparison between the eye of Pecten, the scallop, and the vertebrate eye. This comparison is used as the basis of some far-reaching conclusions, and therefore it becomes important to direct the attention of readers of NATURE to the fact that the example taken is an extremely bad one. Prof. Bergson states that the eye of Pecten agrees in the most minute details with the vertebrate eye. Now there is no resemblance whatever either in structure or development between the two. The only feature possessed in common by both eyes is an inverted retina, and this is by no means unique in the animal kingdom.

University of Liverpool, March I9.

\section{Mersenne's Numbers.}

AT various times NATURE has inserted notices of the successive discoveries in relation to Mersenne's Numbers. In the issue of August 12, x909, Colonel Cunningham's discovery that 228479 was a factor of $2^{p}-\mathrm{I}$ when $p=7 \mathrm{x}$ was announced: the other factor was $10334355^{6} 63637793$, but whether this was a prime or not was left undetermined. The same result was discovered last January by Mr. Ramesam, of Mylapore, Madras, and he subsequently resolved the larger factor into the product of $48544 \mathrm{I} 2 \mathrm{x}$ and $2 \mathrm{I} 2885833$. I think these results may be of interest to some of your readers.

Trinity College, Cambridge, March 23.

W. W. Rouse Ball.

\section{The Electrolytic Transportation of the Active Deposit of Actinium through Pure Water.}

IN the course of some detailed investigations on the conditions of the electrolysis of some radio-active products, I have encountered in the case of actinium the following phenomenon. The products of the active deposit of actinium, though apparently not soluble in water under ordinary conditions, under the action of electric force could be driven from the anode in the state of ions into the water, and eventually transported to the kathode. The experiments were as follows.

The active deposit of actinium was collected on the surface of a platinum plate exposed during some hours as a negative electrode in the emanation of actinium. Immediately after the removal of the plate from the emanation, its activity was measured by means of an electrometer, and the beginning of the curve of its decay was determined. The plate was then immersed in pure, several times distilled water, and formed the anode during the electrolysis of the water. The lxathode was also a platinum plate. The activity of the anode was again measured after the electrolysis. It could be seen that the plate was in certain conditions deprived by the electrolysis of a great part of its activity, especially in the case when a very great electromotive force (220 or 440 volts) was applied. If the current passed through the water during a longer time ( 2 or $3 \mathrm{~min}$.), and the distance between the electrodes was not too great ( 1 or $2 \mathrm{~cm}$.), a great part and often the total activity lost on the anode could be detected on the kathode. The activity of the kathode proceeded not only from actinium $\dot{C}$ and probably actinium $\mathrm{D}$, but also the product 